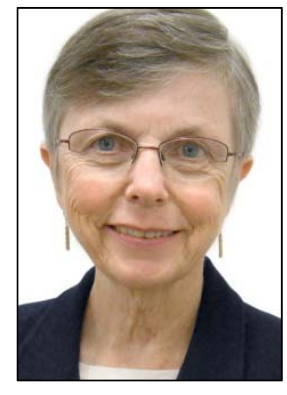

DIGGING DEEPER

Bringing a systems approach to food systems

Kate Clancy

\title{
High-priority research approaches for transforming U.S. food systems
}

Published online September 11, 2013

Citation: Clancy, K. (2013). High priority research approaches for transforming U.S. food systems. Journal of Agriculture, Food Systems, and Community Development, 3(4), 5-7. http://dx.doi.org/10.5304/jafscd.2013.034.021

Copyright (C) 2013 by New Leaf Associates, Inc.

$\mathrm{T}$ he number of agriculture and food research agendas published over the last 25 years would fill multiple shelves - and that's not counting the long lists within each of those agendas. There are so many research needs in every possible area of the food system that the catalog of topics begins to look random. A long-term overall decline in funding, coupled with funders' often narrow preferences and with the academic culture of

Kate Clancy is a food systems consultant, visiting scholar at the Center for a Livable Future, Bloomberg School of Public Health at Johns Hopkins University, and senior fellow at the Minnesota Institute for Sustainable Agriculture. She received her bachelors and doctoral degrees in nutrition at the University of Washington and the University of California Berkeley, respectively. She has studied food systems for over 40 years and has held positions in several universities, the federal government and two nonprofit organizations. Her present interests are regional food systems, food security, agriculture of the middle, and policies at all levels to encourage the development of resilient food systems. freedom to choose one's own research interests, have made food and agricultural research feel chaotic. Priorities and strategies may guide research project choices within some categories, but don't seem to in most. In this context I want to highlight four different approaches and several projects that I believe are very high priority and are necessary to pursue if there is to be a chance of building a sustainable and resilient agrifood system for the future.

Most of these suggestions come from the National Research Council (NRC) publication, Toward Sustainable Agricultural Systems in the 21st Century, published in 2010. (If you haven't read at least some of the report I beseech you to do so.) The report, a follow-up to the NRC report Alternative Agriculture published in 1989, "assesses the scientific evidence for the strengths and weaknesses of different production, marketing, and policy approaches for improving agricultural sustainability and reducing the costs and unintended consequences of agricultural production" (p. vii). The study committee included 15 members 
with expertise in food production and agribusiness; crop, soil, and horticultural sciences; water -use and water- quality science; farming systems and agroecology; agricultural economics and social science; and federal farm, trade, international development, environmental, and regulatory policies. Two of the committee members were farmers (p. vii).

First, the committee urges the research community to find a way to structure inquiries and approaches so that while incremental research continues, the strongest emphasis is placed on transformative research.

These are projects that show the way to systemic changes that are quite different from the present and dominant system. Examples include organic and managed intensive grazing production systems; values-based whole supply chain development; and sustainable retail structures and supply chains that lower the vulnerability toward food insecurity in low-income areas.

Second, the committee argues, researchers need to identify and examine systems characteristics that will increase adaptability and resilience. The latter is defined by the committee as "the capacity of the system to absorb shocks or perturbations and still retain and further develop the same fundamental structure functioning and feedbacks" (Chapin et al., 2009, in NRC report, p. 26). It strikes me that at this time we don't have a good idea what resilient food and agricultural sectors look like. Research describing options that would make a system or sector resilient across the supply chain would be a major contribution to our understanding of where the U.S. food system is most vulnerable to shocks such as drought, economic downturns, or loss of biodiversity, and in the types of restructuring that can bring greater resiliency. It is systems research that is called for not piecemeal efforts. Projects need to explore the interdependencies between the biophysical and socio-economic aspects of food and farming systems. The proper scale needs to be addressed and people need to look carefully at how different scales - local, regional, national, and global must interact.

Third, the NRC committee is a strong advocate for programs that take a landscape approach to the design of agricultural ecosystems, which "maximize synergies, enhance resilience, and inform what policies would be useful in influencing collective action" (NRC report, p. 11). This is research that takes into account large landscapes such as watersheds and multistate regions, not individual farms or farmers. I would add that the need for a broader view applies as well to the rest of the supply-chain members, such as processors and retailers and their networks.

To me one of the benefits of a landscape approach is bringing more attention to farmland preservation. I don't think that food system researchers are devoting enough effort to this issue. How can the U.S. possibly meet food demands, even accounting for food produced in cities in the future, if it doesn't retain its remaining farmland and figure out how to provide access to it by young and beginning farmers? The political, social, and behavioral aspects of this task are daunting. What kind of incentives, regulations, and institutions could encourage locales to be more vigilant and creative in partnership with planners and decisionmakers at higher scales - multicounty, state, multistate - in order to guarantee future food security for the country? We need models, best practices, and guidance to accomplish this. These will require multidisciplinary research involving planners, political scientists, and food systems experts working with farmers, developers, and politicians.

A fourth priority research area is investigating new steps that can be taken to support more effective policy-making and "assess the full impacts of current and proposed policy frameworks" (NRC 
report, p. 13) — with regard to much larger and appropriate levels of funding for agrifood research as well as a wide range of needed agriculture and food program changes. Given how extensive this problem is, it is discouraging that there is still so little funding for research on policy options and outcomes. The unwillingness by governments and many foundations to fund policy research has always seemed counterproductive and shortsighted to me and this is indeed proving to be the case.

An obvious final priority (mine, not the committee's) is to ask who is going to carry out this transformative, multidisciplinary, landscape-level policy research in the near and far future. Most likely it is students who are learning about systems science and systems research and are participating in extensive transdisciplinary food systems research projects. At this point the ranks of those students are much too small across the country. Advocates need to call on funders, deans, and advisors to overhaul curricula and research training to meet these new needs.

I join the Committee in expressing a sense of urgency toward the entire agrifood research project. In its words, "agricultural production will have to substantially accelerate progress towards sustainability goals" (emphasis added; NRC report, p. 5). And I would state again that not just the production sector but all parts of supply chains need to hurry on up. Given the paucity of research funds and the serious big picture needs and implications described here, I believe a realistic and important argument can be made for funders and researchers to expend more effort in prioritizing their research choices, in pursuing transdisciplinary projects, and in focusing on resilience.

I want to thank Kathy Ruhf of Land for Good for her contributions - especially to the section on farmland retention and access. Look for her paper, "Access to Farmland: A Systems Change Perspective," being published in the fall issue of JAFSCD.

\section{References}

Committee on Twenty-First Century Systems Agriculture \& National Research Council. (2010). Toward Sustainable Agricultural Systems in the 21st Century. Washington, D.C.: National Academies Press. 